\title{
Mode Locking of the Hermite-Gaussian Modes of a Nanolaser
}

\author{
Yifan Sun, ${ }^{1}$ Sylvain Combrié, ${ }^{2}$ Fabien Bretenaker@, ${ }^{1,3}$ and Alfredo De Rossi ${ }^{2}$ \\ ${ }^{1}$ Laboratoire Aimé Cotton, Université Paris-Sud, ENS Paris-Saclay, CNRS, Université Paris-Saclay, 91405 Orsay Cedex, France \\ ${ }^{2}$ Thales Research and Technology, 91120 Palaiseau, France \\ ${ }^{3}$ Light and Matter Physics Group, Raman Research Institute, Bangalore 560080, India
}

(Received 2 August 2019; published 3 December 2019)

\begin{abstract}
Mode locking is predicted in a nanolaser cavity forming an effective photonic harmonic potential. The cavity is substantially more compact than a Fabry-Perot resonator with a comparable pulsing period, which is here controlled by the potential. In the limit of instantaneous gain and absorption saturation, mode locking corresponds to a stable dissipative soliton, which is very well approximated by the coherent state of a quantum mechanical harmonic oscillator. This property is robust against noninstantaneous material response and nonzero phase-intensity coupling.
\end{abstract}

DOI: 10.1103/PhysRevLett.123.233901

Mode-locked (ML) diode lasers are compact sources of short pulses with countless applications [1]. The growing relevance of short-distance and on-chip optical communications for future computers [2] has stimulated the emergence of novel ultracompact sources meeting severe energy requirements [3]. In this respect, a milestone is the achievement of microwatt power consumption with very competitive wall-plug efficiency in nanolaser diodes [4]. A crucial role here is played by the photonic crystal (PC) confining light within a very small volume. These optical sources can be directly modulated [5]. Related to ML is self-pulsing, recently achieved by coupling a PC cavity with a PC waveguide Fabry-Perot resonator [6]. The aim of this Letter is to introduce a new approach to ML in nanocavities, whereby the eigenfrequencies are engineered to become evenly spaced, as recently demonstrated experimentally [7]. For that purpose, we introduce a model describing the nonlinear laser dynamical behavior and look for the conditions for robust mode locking. In particular, we show how the peculiarities of the mode-locking process in such nanolasers permit us to overcome the usual trade-off between laser size and pulsing period. Moreover, we show that the considered ML laser behaves according to the coherent state of a quantum harmonic oscillator, thus bridging the gap between nonlinear nanophotonics and quantum optics.

In a Fabry-Perot cavity [Fig. 1(a)] and similar resonators (racetracks, whispering gallery modes,...), resonance results from constructive interference of propagating waves. The round-trip time $T$ is directly related to the resonator length $L$ through $T=2 L / v_{g}$, where $v_{g}$ is the group velocity. If modes are locked, the cavity round-trip time materializes into a pulse propagating back and forth inside the cavity, suggesting a straight mechanical analogy with a free particle bouncing between two barriers. Locking both the longitudinal and transverse modes of a fiber laser has been considered very recently in order to control the spatiotemporal profile of the emitted light [8].

Here, we consider the completely different situation of a photonic resonator made of a metamaterial with effective parabolic dispersion $\omega_{k k}$ and parabolic effective photonic potential $V(x)$ [see Fig. 1(b)], $x$ denoting the spatial degree of freedom. A laser based on such a resonator cannot be described by conventional ML laser theory $[9,10]$. A straightforward implementation of this metamaterial is a quasiperiodic photonic structure. Indeed, Sipe et al. [11,12] have theoretically demonstrated, using the multiple scales method and Floquet-Bloch theory, that the dynamical behavior of the slowly varying field envelope $A(x, t)$ of the Bloch waves in a nonlinear medium with periodic dielectric structure is governed by the nonlinear Schrödinger equation. The effective parabolic dispersion $\omega_{k k}=\partial^{2} \omega / \partial k^{2}$ is the second order dispersion of the normal Bloch mode. In the simplest periodic structure (a)

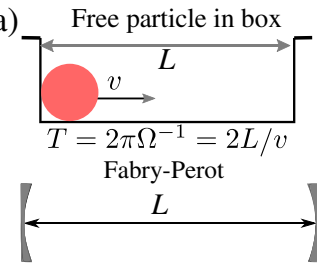

(c)

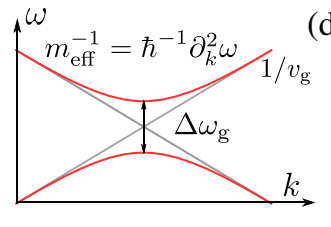

(b)

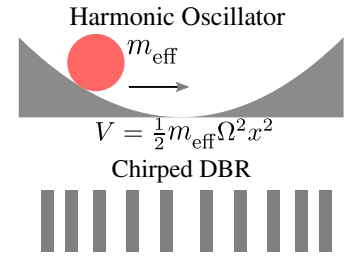

(d)

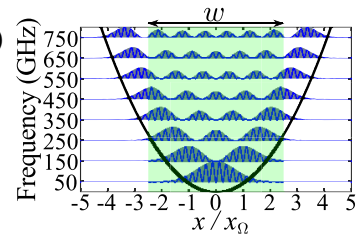

FIG. 1. Mechanical analogy of (a) Fabry-Perot resonator and (b) harmonic oscillator implemented in a chirped DBR; (c) Parabolic dispersion in a DBR; (d) Hermite-Gaussian modes in a DBR with parabolic potential. Green area: gain and absorber region. 
model [Fig. 1(b)], the coupled mode theory of a distributed Bragg reflector (DBR) [13] in a waveguide with group velocity $v_{g}$ relates the dispersion to the width of the photonic band gap $\Delta \omega_{g}$ through $\omega_{k k}=2 v_{g}^{2} / \Delta \omega_{g}$. In general, a PC allows control of the dispersion [14] and in most cases, the band edge is located at a high-symmetry point in the reciprocal space. Consequently, the dispersion is symmetric and third order dispersion is zero. As higher order dispersion could in principle be controlled, this approximation is well justified within the spectral domain of interest (see the detailed discussion in the Supplemental Material [15]).

The Gross-Pitaevskii equation (GPE) is constructed by adding a potential $V(x)$ to the nonlinear Schrödinger equation resulting in a linear confinement [16]. This can be obtained by building a dielectric guiding nanostructure with one of its parameters, for example the period $a$ of the confining holes, slowly varying with $x$ in a parabolic manner: $a(x)=a_{0}+\varsigma x^{2}$. Then, in the limit of small changes of $a$, it can be conjectured that the normal modes and $\omega_{k k}$ are not changed, and the GPE still holds. The only modification is that the local change of $a$ induces a frequency offset of the dispersion $V(x) \propto\left[a(x)^{-1}-a_{0}^{-1}\right] \propto$ $-\varsigma x^{2}$ with $\varsigma=\left(-\Omega^{2} / 2 \omega_{k k}\right)\left(a_{0} / \omega_{c}\right)$ where $\omega_{c}$ is the edge of the photonic band. Hence, a chirped periodic dielectric nanostructure results in a harmonic potential $V(x)$ for the field envelope of the normal modes near the band edge. A multimode high- $Q$ optical resonator with an effective harmonic parabolic potential has been experimentally demonstrated [7,17]. Such a structure is therefore described by the linear and nondissipative limit of the GPE equation, written here in a form such that the dispersion $\omega_{k k}$ also appears in the potential term

$$
i \frac{\partial A}{\partial t}+\frac{1}{2} \omega_{k k} \frac{\partial^{2} A}{\partial x^{2}}-\frac{1}{2} \frac{\Omega^{2}}{\omega_{k k}} x^{2} A=0
$$

This equation is strictly equivalent to quantum mechanical harmonic oscillator and the envelope is described by a linear superposition of the Hermite-Gaussian eigenmodes $\Psi_{n}(x)$ with equally spaced eigenfrequencies $\omega_{n}=$ $(n+1 / 2) \Omega+\omega_{c}$ :

$$
A(x, t)=\sum_{n=0}^{\infty} C_{n}(t) e^{-i \omega_{n} t} \Psi_{n}(x) .
$$

If the modes could be phase locked such as to form the optical equivalent of the coherent state of the quantum harmonic oscillator, then the wave packet would be described by a Gaussian pulse with velocity and position obeying a sinusoidal evolution without deformation and oscillation period $T=2 \pi \Omega^{-1}$ corresponding to the frequency separation $\Omega / 2 \pi$. The case of the effective harmonic potential is therefore of particular interest in the context of laser.

Hermite-Gaussian modes are very different from plane waves as they are spatially inhomogeneous. This has a profound implication in their nonlinear interaction. Moreover, contrary to the Fabry-Perot cavity, the oscillation period does not depend on the size of the oscillator but on the effective photon mass $m_{\text {eff }}^{-1}=\hbar^{-1} \partial_{k}^{2} \omega \equiv \hbar^{-1} \omega_{k k}$ of the particle and the stiffness of the potential, which can be expressed as $V(x)=\frac{1}{2} m_{\mathrm{eff}} \Omega^{2} x^{2} / \hbar$. The analogy is sketched in Fig. 1. As the fractional photonic band gap of PC cavities is typically $\Delta \omega / \omega \approx 20 \%$ [18] and the group velocity in semiconductor waveguides is about $c_{0} / 4$, the model of a distributed Bragg reflector leads to an order of magnitude estimate of the dispersion $\omega_{k k}=45 \mathrm{~m}^{2} \mathrm{~s}^{-1}$. Setting $\Omega / 2 \pi=100 \mathrm{GHz}$ leads to a length scale for the Hermite-Gaussian modes $x_{\Omega}=\sqrt{\omega_{k k} / \Omega}$, which is equal to $8.4 \mu \mathrm{m}$. The full width at half maximum of the fundamental mode is then $2 \sqrt{\ln 2} x_{\Omega} \simeq 14 \mu \mathrm{m}$ and the size of a cavity containing $N$ modes scales as $2 \sqrt{N} x_{\Omega}$ [Fig. 1(d)].

We investigate now whether such a comb of modes can passively mode lock when this harmonic resonator contains or is hybridized to an active medium providing gain and saturable absorption. The laser nonlinear dynamical behavior is then described by the modified GPE:

$$
i \frac{\partial A}{\partial t}+\frac{1}{2} \omega_{k k} \frac{\partial^{2} A}{\partial x^{2}}-V(x) A-i H_{1}\left(|A|^{2}\right) A=0 .
$$

$H_{1}$ holds for the dissipative terms and the nonlinear terms that depend on $|A|^{2}$ :

$H_{1}=\frac{1}{2} g(x, t)\left(1-i \alpha_{g}\right)-\frac{1}{2} a(x, t)\left(1-i \alpha_{a}\right)-\frac{1}{2} \gamma_{0}$,

where $g(x, t)$ and $a(x, t)$ are the time and space dependent gain and saturable loss coefficients associated with the Henry factors $\alpha_{g}$ and $\alpha_{a}$, respectively, and $\gamma_{0}$ holds for the intrinsic losses. Here, the gain is assumed spectrally flat, which is realistic for quantum well and quantum dot active materials and a signal bandwidth about $1 \mathrm{THz}$.

For simplicity, we first consider the case where saturation of the gain and losses is instantaneous, leading to

$$
g(x, t)=g_{0}(x) /\left(1+\frac{|A(x, t)|^{2}}{I_{\mathrm{sat}, g}}\right),
$$

with a similar expression for $a(x, t)$. Here, $I_{\text {sat, } g}$ and $I_{\text {sat }, a}$ are the saturation intensities for the unsaturated gain and absorption coefficients $g_{0}$ and $a_{0}$, respectively. We can then numerically solve Eq. (3) for different values of the parameters, and for different values of the widths of the windows into which $g_{0}$ and $a_{0}$ are supposed to be homogeneous. A first example is given in Fig. 2, which was obtained when the gain and saturable absorber share 

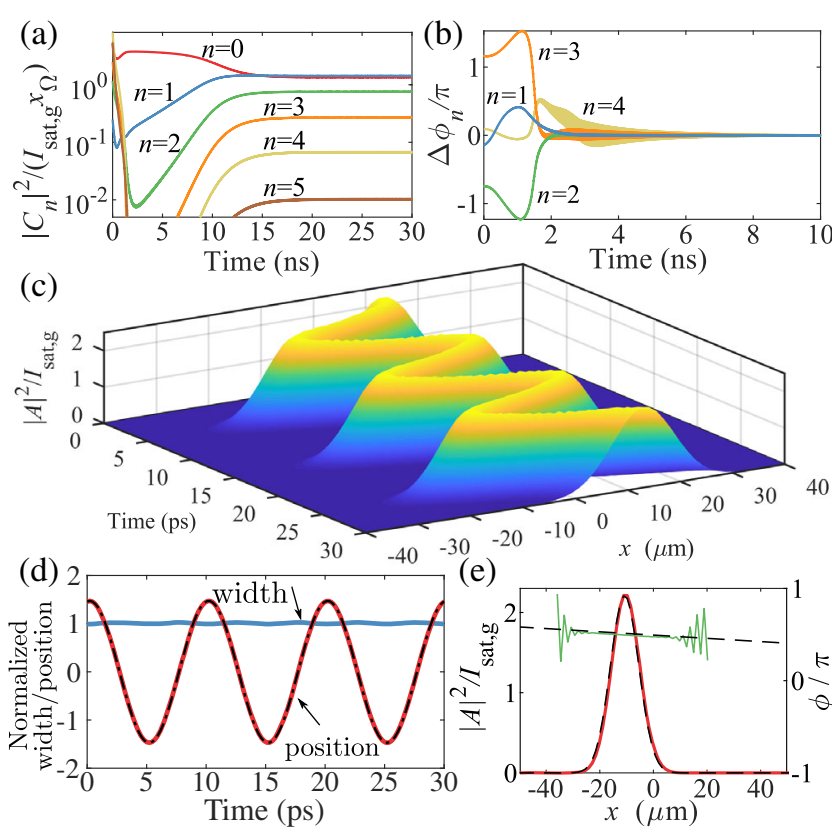

FIG. 2. Laser behavior for instantaneous gain and absorber saturation with common widths equal to $5 x_{\Omega}$. (a),(b) Transient evolutions of (a) normalized intensities and (b) relative phases between the modes after the simulation is started from random mode amplitudes. (c) Evolution of the intracavity intensity in steady-state regime. (d) Positions of the coherent state (black dashed line) and the soliton (solid red line) and soliton width (solid cyan line) normalized to $x_{\Omega}$. (e) Amplitude (left axis, solid red line) and phase (right axis, solid green line) of the soliton at a fixed time and corresponding coherent state (dashed line) with amplitude $2.2 I_{g}$.

the same region of width $w=5 x_{\Omega}$ [see Fig. 1(d)]. The parameter values are $\gamma_{0}=10^{10} \mathrm{~s}^{-1}, r_{g}=g_{0} / \gamma_{0}=5.5$, $r_{a}=a_{0} / \gamma_{0}=9$, and $R_{I}=I_{\mathrm{sat}, g} / I_{\mathrm{sat}, a}=5$. Such a value of the internal (nonsaturable) losses $\gamma_{0}$ is small, but feasible for a semiconductor cavity [19]. We also suppose here that $\alpha_{g}=\alpha_{a}=0$. Equation (3) is solved starting from random initial conditions, and the behaviors of the different modes are obtained by projecting $A(x, t)$ on the corresponding $\Psi_{n}(x)$. Figure 2(a) shows the evolution of the normalized intensities of the first six modes, which are the only ones that reach significant steady-state intensities after a few tens of nanoseconds. To determine whether this multimode behavior corresponds to ML operation, we plot the evolutions of the relative phases $\Delta \phi_{n}=2 \phi_{n}-\phi_{n-1}-\phi_{n+1}$ between the modes for $n=1, \ldots, 4$ in Fig. 2(b). Here, $\phi_{n}$ is the argument of the mode expansion coefficient $C_{n}$ of Eq. (2). One can clearly see that after less than $10 \mathrm{~ns}$ all the lasing modes are phase locked. Once steady state is reached, i.e., after about $30 \mathrm{~ns}$, the laser behavior is shown in Fig. 2(c).

Closer inspection of the spatiotemporal behavior reveals a wobbling soliton [20], described by sinusoidally varying width and position. These quantities are plotted, normalized to $x_{\Omega}$, in Fig. 2(d) and coincide almost exactly, except
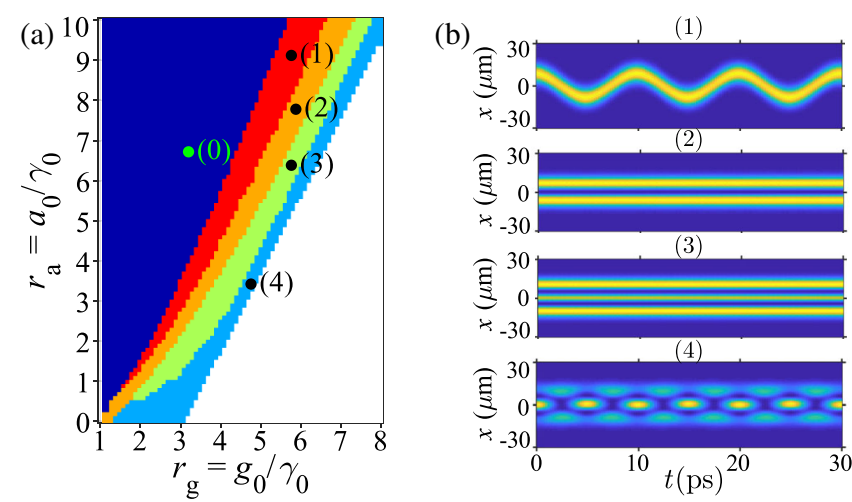

FIG. 3. (a) Phase diagram: different steady-state regimes vs unsaturated gain and absorption normalized to $\gamma_{0}$. White hatched region: different unlocked mutimode regimes. (b) Corresponding false color plots of the laser intensity spatial distribution vs time, for different regimes: (0) below threshold; (1) solitonlike pulse; (2) mode $n=1$ alone; (3) mode $n=2$ alone; (4) simultaneous oscillation of modes $n=0$ and $n=2$.

for small residual oscillation $(<1 \%)$, with the superposition of the linear eigenstates [Eq. (2)], describing the coherent state of the harmonic oscillator in quantum mechanics. This is further apparent in Fig. 2(e) where the amplitude and phase of the field at a fixed time are compared with the coherent state. More detailed analysis is given in the Supplemental Material [15].

The nonlinear laser behavior undergoes bifurcations separating different possible behaviors. Colors in Fig. 3(a) represent the regions, in the $\left\{r_{g}=g_{0} / \gamma_{0}, r_{a}=a_{0} / \gamma_{0}\right\}$ plane, where different steady-state behaviors dominate. The solitonlike [20] pulsed operation of Figs. 2(c)-2(e) does not only require a sufficient amount of gain, but also a sufficient amount of saturable absorption. Our choice for $R_{I}=$ $I_{\mathrm{sat}, g} / I_{\mathrm{sat}, a}=5$ larger than 1 is also extremely important to obtain this behavior.

Although different shapes can be imagined for the gain and absorber, we consider only identical homogeneous gain and saturable absorption windows of width $w$ centered on the potential minimum [green area in Fig. 1(d)]. To investigate the influence of $w$, we launch the simulation 40 times, starting from random initial fields, for each value of $w$ ranging from 0 to $6 x_{\Omega}$. The system exhibits multistability: it can reach different steady-state regimes for a given set of parameters, depending on the initial values. To gain some statistical insight into this multiattractor behavior, Fig. 4(a) displays the occurrences of each regime vs $w$. For $w$ increasing from 0 to $3.2 x_{\Omega}$, the laser is successively below threshold, in single-mode regime, and finally emits the soliton solution of Fig. 2(c). Interestingly, in the range $3.2 x_{\Omega} \lesssim w \lesssim 3.9 x_{\Omega}$, soliton emission is the only stable solution. In this example, multistability happens for values of $w$ larger than $3.9 x_{\Omega}$. For example, for $w=6 x_{\Omega}$, Fig. 4(a) shows that the laser dynamical behavior can fall into three different stable ML regimes, in which one, two, or three 

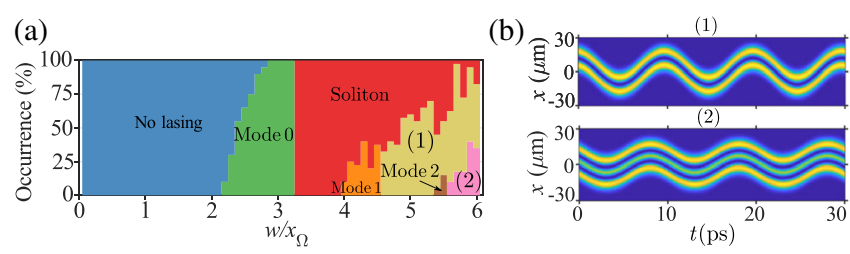

FIG. 4. (a) Percentage of occurrences of the different regimes, when the simulation is run 40 times with random initial conditions for each value of the gain and saturable absorption window width $w$. Other parameters are $R_{I}=5, g_{0}=5.5 \gamma_{0}$, $a_{0}=9 \gamma_{0}$. (b) False color plot of laser intensity vs $x$ and $t$ in two examples of regimes labeled (1) and (2) in (a), obtained for $w=6 x_{\Omega}$. They, respectively, correspond to oscillation of two or three pulses inside the cavity.

pulses oscillate inside the cavity. Figure 4(b) gives examples of these two last behaviors. The numerical study reveals that the width $w$ of the gain region is the most important parameter determining the number of locked modes and therefore the spatial amplitude of the pulse oscillation, which is close to $w$.

Real semiconductor gain and saturable absorption media have typical response times $\tau_{g}$ and $\tau_{a}$ ranging from the picosecond to the nanosecond domain [21], i.e., not always negligible compared with the photon lifetime in nanocavities or with picosecond pulse durations as in Fig. 2(c). To investigate the role of such finite lifetimes, we have solved the modified GPE Eq. (3) with Eq (5) replaced by

$$
\frac{\partial g(x, t)}{\partial t}=-\frac{g(x, t)-g_{0}(x)}{\tau_{g}}-\frac{|A(x, t)|^{2}}{\tau_{g} I_{\text {sat }, g}} g(x, t),
$$

with a similar equation for $a(x, t)$. The values of the lifetimes $\tau_{g}=1 \mathrm{~ns}$ and $\tau_{a}=10 \mathrm{ps}[22,23]$ we choose are those typically mentioned in the literature for InP quantum well lasers [24]. Surface recombination has been recently improved in nanostructured lasers owing to advanced passivation techniques such that the carrier lifetime is a few nanoseconds [4]. We also take a ratio of the saturation energies $R_{E}=I_{\text {sat, }, g} \tau_{g} / I_{\text {sat }, a} \tau_{a}=25$ from the literature [23]. This leads to the results of Fig. 5, computed with $\alpha_{g}=\alpha_{a}=0$. The phase diagram of Fig. 5(a) exhibits new regimes, such as passively $Q$-switched operation, either in unlocked (point labeled 1) or $Q$-switched ML regime (point labeled 2). However, with a proper choice of $a_{0}$ and $g_{0}$, one can still obtain cw passively ML operation, as evidenced by the red region of Fig. 5(a). Figures 5(b)-5(d) show one example of such a behavior. The solution is again very close to a coherent state, although the shape of the pulse gets slightly distorted close to its turning point [see Figs. 5(b) and 5(c)]. Between these points, Fig. 5(d) shows that the spatial pulse shape is quite well adjusted by a coherent state. Further discussions of discrepancies with respect to a perfect coherent state are provided in the Supplemental Material [15]. Moreover, a simulation given in Sec. V of the
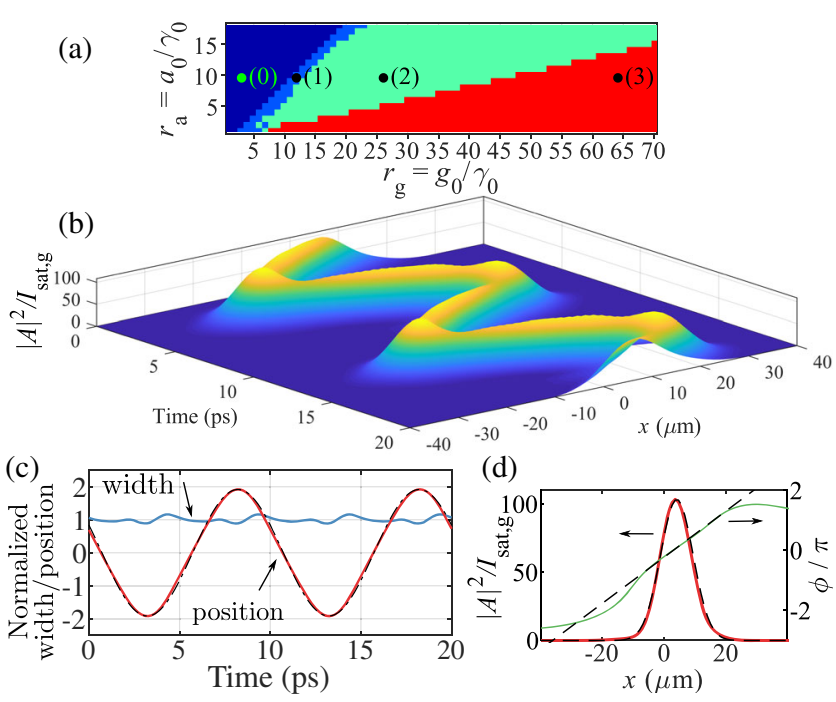

FIG. 5. Laser behavior for slow gain and absorber saturations. (a) Phase diagram: different steady-state regimes vs unsaturated gain and absorption normalized to $\gamma_{0}:(0)$ below threshold; (1) single-mode $Q$-switched operation; (2) $Q$-switched $\mathrm{ML}$ operation; (3) continuous-wave mode locking. (b),(c),(d) Stable ML behavior. Same as Figs. 2(c)-2(e) corresponding to $r_{g}=70$ and $r_{a}=10$ in area number (3).

Supplemental Material [15] indicates that the multistability of Fig. 4 seems to disappear for finite response times, which is positive for practical applications of such nanolasers.

A nonzero Henry factor, coupling the phase and intensity variations through carrier dynamics, is known to be a source of instability for passive ML [25]. However, the laser bifurcation diagram shows that ML, similar to Fig. 5, can still be obtained with nonzero values of $\alpha_{g}$ and $\alpha_{a}$ at the cost of an increase of the pumping (see the Supplemental Material [15]).

Experimental implementation of the harmonic cavity nanolaser can be envisaged as follows. We consider a photonic crystal made of InP and containing InGaAsP quantum wells to provide enough gain $[5,26]$ for lasing. It has also been demonstrated that the laser can be operated well above threshold before any saturation occurs $\left(I>5 I_{\text {th }}\right.$ [4]), meaning that the unsaturated gain can exceed many times the nonsaturable losses. A saturable absorber can be implemented in many ways, for instance like in Ref. [27]. The harmonic photonic potential is obtained through a suitable design, for instance using a bichromatic lattice as an alternative to a chirped period [7]. As shown in Fig. 5(a), mode locking could be observed with a gain exceeding saturable losses by a factor close to 5 , which is achievable since the $Q$ factor of InP photonic crystals is about $10^{5}$ [28]. The large photonic band gap of these structures results in a large effective photon mass such that the typical cavity size for $100 \mathrm{GHz}$ period would be less than $100 \mu \mathrm{m}$. Thus, the essential requirements for building an extremely compact ML integrated nanolaser are met by the current state-of-theart of nanolaser technology. Balanced extraction of power 
from all the locked modes could be achieved with a geometry like that in [4].

In conclusion, a novel concept for ML in ultracompact semiconductor lasers has been proposed, based on a harmonic potential to confine light. This maps the optical cavity into a quantum mechanical harmonic oscillator, with evenly spaced eigenfrequencies, an essential requirement for ML. The nonlinear behavior is described by the GrossPitaevskii equation with a parabolic potential and nonlinear terms describing gain and absorption. ML occurs with Hermite-Gaussian modes, which are very different from waves of usual resonators, as they are stationary modes with a strongly inhomogeneous spatial distribution of energy. Provided that saturable gain and absorption overlap with all the modes, ML occurs over a broad area in the phase space, corresponding to the emergence of dissipative soliton and multisoliton solutions. In the limit of instantaneous absorption and gain saturation, the dissipative soliton is well described by the coherent state of a quantum mechanical oscillator, namely a Gaussian envelope oscillating without deformation. ML period is controlled by the design of the photonic potential, and not by the cavity length. For a fixed ML period, here $10 \mathrm{ps}$, the linear size of our cavity is $80 \mu \mathrm{m}$, about five times more compact than for a Fabry-Perot laser made with the same material. Finally, slow absorption or gain response still allows ML and most features of the coherent state are retained. Thus, the concept of ML based on Hermite-Gaussian modes in photonic nanostructures could solve the long-standing problem of miniature periodic pulsed sources [26,29]. Moreover, the laser behaving like a quantum harmonic oscillator makes a link between nonlinear nanophotonics and quantum optics, opening the way to interesting ramifications regarding quantum photon statistics in such ML nanolasers. Furthermore, analyzing the full bifurcation diagram [30] is interesting from the viewpoint of nonlinear dynamical systems theory.

Work supported by the Direction Générale de l'Armement (LASAGNE, ANR-16-ASTR-0010-03), the "Investissements d'Avenir" program (CONDOR, ANR10-LABX-0035), European Union's Horizon 2020 program (Fun-COMP, Grant Agreement No. 780848) and performed in the framework of the joint research lab between TRT and LAC.

[1] E. Rafailov, M. A. Cataluna, and W. Sibbett, Mode-locked quantum-dot lasers, Nat. Photonics 1, 395 (2007).

[2] C. Sun et al., Single-chip microprocessor that communicates directly using light, Nature (London) 528, 534 (2015).

[3] D. A. B. Miller, Device requirements for optical interconnects to silicon chips, Proc. IEEE 97, 1166 (2009).

[4] G. Crosnier, D. Sanchez, S. Bouchoule, P. Monnier, G. Beaudoin, I. Sagnes, R. Raj, and F. Raineri, Hybrid indium phosphide-on-silicon nanolaser diode, Nat. Photonics 11, 297 (2017).

[5] S. Matsuo, A. Shinya, T. Kakitsuka, K. Nozaki, T. Segawa, T. Sato, Y. Kawaguchi, and M. Notomi, High-speed ultracompact buried heterostructure photonic-crystal laser with $13 \mathrm{fJ}$ of energy consumed per bit transmitted, Nat. Photonics 4, 648 (2010).

[6] Y. Yu, W. Xue, E. Semenova, K. Yvind, and J. Mørk, Demonstration of a self-pulsing photonic crystal Fano laser, Nat. Photonics 11, 81 (2017).

[7] S. Combrié, G. Lehoucq, G. Moille, A. Martin, and A. De Rossi, Comb of high- $Q$ resonances in a compact photonic cavity, Laser Photonics Rev. 11, 1700099 (2017).

[8] L. G. Wright, D. N. Christodoulides, and F. W. Wise, Spatiotemporal mode-locking in multimode fiber lasers, Science 358, 94 (2017).

[9] H. A. Haus, Mode-Locking of lasers, IEEE J. Sel. Top. Quantum Electron. 6, 1173 (2000).

[10] J. Javaloyes and S. Balle, Mode-locking in semiconductor Fabry-Perot lasers, IEEE J. Quantum Electron. 46, 1023 (2010).

[11] J. E. Sipe and H. G. Winful, Nonlinear Schrödinger solitons in a periodic structure, Opt. Lett. 13, 132 (1988).

[12] C. M. de Sterke and J. E. Sipe, Envelope-function approach for the electrodynamics of nonlinear periodic structures, Phys. Rev. A 38, 5149 (1988).

[13] A. Yariv, Coupled-mode theory for guided-wave optics, IEEE J. Quantum Electron. 9, 919 (1973).

[14] M. Notomi, K. Yamada, A. Shinya, J. Takahashi, C. Takahashi, and I. Yokohama, Extremely Large GroupVelocity Dispersion of Line-Defect Waveguides in Photonic Crystal Slabs, Phys. Rev. Lett. 87, 253902 (2001).

[15] See Supplemental Material at http://link.aps.org/ supplemental/10.1103/PhysRevLett.123.233901 for further details on dispersion in the PC cavity, comparison of the soliton solution with a coherent state, numerical methods, and the influence of the Henry factor and the finite lifetimes of the absorber and the gain medium.

[16] M. C. F. Dobbelaar, S. Greveling, and D. van Oosten, Large area photonic crystal cavities: A local density approach, Opt. Express 23, 7481 (2015).

[17] S. V. Suchkov, M. Sumetsky, and A. A. Sukhorukov, Frequency comb generation in SNAP bottle resonators, Opt. Lett. 42, 2149 (2017).

[18] J. S. Foresi, P. R. Villeneuve, J. Ferrera, E. R. Thoen, G. Steinmeyer, S. Fan, J. D. Joannopoulos, L. C. Kimerling, H. I. Smith, and E. P. Ippen, Photonic-bandgap microcavities in optical waveguides, Nature (London) 390, 143 (1997).

[19] C. T. Santis, S. T. Steger, Y. Vilenchik, A. Vasilyev, and A. Yariv, High-coherence semiconductor lasers based on integral high- $Q$ resonators in hybrid Si/III-V platforms, Proc. Natl. Acad. Sci. U.S.A. 111, 2879 (2014).

[20] P. Grelu and N.Akhmediev, Dissipative solitons for modelocked lasers, Nat. Photonics 6, 84 (2012).

[21] A. Mecozzi and J. Mørk, Saturation effects in nondegenerate four-wave mixing between short optical pulses in semiconductor laser amplifiers, IEEE J. Sel. Top. Quantum Electron. 3, 1190 (1997). 
[22] A. G. Vladimirov, A.S. Pimenov, and D. Rachinskii, Numerical study of dynamical regimes in a monolithic passively mode-locked semiconductor laser, IEEE J. Quantum Electron. 45, 462 (2009).

[23] M. Heuck, S. Blaaberg, and J. Mørk, Theory of passively mode-locked photonic crystal semiconductor lasers, Opt. Express 18, 18003 (2010).

[24] D. J. Jones, L. M. Zhang, J. E. Carroll, and D. D. Marcenac, Dynamics of monolithic passively mode-locked semiconductor lasers, IEEE J. Quantum Electron. 31, 1051 (1995).

[25] A. G. Vladimirov and D. Turaev, Model for passive mode locking in semiconductor lasers, Phys. Rev. A 72, 033808 (2005).

[26] Y. Yu, W. Xue, E. Semenova, K. Yvind, and J. Mørk, Demonstration of a self-pulsing photonic crystal fano laser, Nat. Photonics 11, 81 (2017).
[27] S. Barbay, R. Kuszelewicz, and A. M. Yacomotti, Excitability in a semiconductor laser with saturable absorber, Opt. Lett. 36, 4476 (2011).

[28] G. Crosnier, D. Sanchez, A. Bazin, P. Monnier, S. Bouchoule, R. Braive, G. Beaudoin, I. Sagnes, R. Raj, and F. Raineri, High $Q$ factor InP photonic crystal nanobeam cavities on silicon wire waveguides, Opt. Lett. 41, 579 (2016).

[29] K. Takeda, T. Sato, A. Shinya, K. Nozaki, W. Kobayashi, H. Taniyama, M. Notomi, K. Hasebe, T. Kakitsuka, and S. Matsuo, Few-fJ/bit data transmissions using directly modulated lambda-scale embedded active region photonic-crystal lasers, Nat. Photonics 7, 569 (2013).

[30] S. V. Gurevich, C. Schelte, and J. Javaloyes, Impact of highorder effects on soliton explosions in the complex cubicquintic Ginzburg-Landau equation, Phys. Rev. A 99, 061803(R) (2019). 